\title{
Enhancing the Reduction Potential of Quinones via Complex Formation
}

\author{
Binod Nepal and Steve Scheiner* \\ Department of Chemistry and Biochemistry \\ Utah State University \\ Logan UT 84322-0300
}

\begin{abstract}
Quantum calculations are used to study the manner in which quinones interact with proton-donating molecules. For neutral donors, a stacked geometry is favored over a H-bond structure. The former is stabilized by charge transfers from the $\mathrm{N}$ or $\mathrm{O}$ lone pairs to the quinone's $\pi^{*}$ orbitals. Following the addition of an electron to the quinone, the radical anion forms strong H-bonded complexes with the various donors. The presence of the donor enhances the electron affinity of the quinone. This enhancement is on the order of $15 \mathrm{kcal} / \mathrm{mol}$ for neutral donors, but up to as much as $85 \mathrm{kcal} / \mathrm{mol}$ for a cationic donor. The increase in electron affinity is larger for electron-rich quinones, than for their electron-deficient counterparts, containing halogen substituents. Similar trends are in evidence when the systems are immersed in aqueous solvent.

*email: steve.scheiner@usu.edu
\end{abstract}

keywords: M06-2X; NBO; electron affinity; stacked structure; H-bond 


\section{Introduction}

Quinones represent an important class of organic compounds which are present in many biologically active sites. For example, plastoquinone and phylloquinone act as the electron accepters in the electron transport chain in photosynthesis. ${ }^{1}$ Ubiquinone is the electron acceptor in aerobic respiration. ${ }^{2,3}$ Several quinone compounds have been found to have anticancer, antibacterial ${ }^{4-6}$ and antifungal activity. ${ }^{7}$ Similarly, quinone compounds have a wide range of application in synthetic chemistry, catalysis, and electrochemistry. ${ }^{8-14}$ Active research continues to assess the usefulness of quinone in lithium- $\mathrm{O}_{2}$ batteries. 15-17 Quinones are very good oxidizing agents and can undergo one or two electron reduction, forming monoanion and dianion radical, respectively, depending on the conditions. This electron transfer to the quinones can be coupled with proton transfer. ${ }^{18,19}$

A number of studies, both experimental and theoretical, have shown that the redox potential of quinones can be increased by suitable $\mathrm{H}$-bond (HB) donor systems, which assist the electron transfer by stabilizing the resulting radical anion by H-bonding. ${ }^{20-24}$ Depending on the solvent media and the $\mathrm{pK}_{\mathrm{a}}$ of the HB donor, proton donation may accompany the electron transfer. Various types of HB donors including charged, neutral single-H donor, bidentate etc. have been exploited to activate the oxidizing activity of the quinone compounds. ${ }^{20,25-27}$ Interestingly, a number of studies indicate that the HB donors might increase the oxidizing strength of electron-rich quinones but not that much for electron deficient quinones. Very recently, Nocera and Jacobsen's research group published an intriguing article ${ }^{20}$ which showed that dicationic HB donors can strongly activate electron-deficient quinones like chloranil, and that the rate of electron transfer can be increased by more than 12 orders of magnitude when coupled with a suitable dicationic H-bond donor. Their study also revealed that an equally acidic HB donor can yield completely different results based on the electrostatic component of H-bond. These cationic donors display greater activation role in electron-deficient quinones.

The kinetics of the electron transfer reaction can be explained in terms of Marcus theory. ${ }^{28}$ The rate of electron transfer is dependent on both the free energy change $\Delta \mathrm{G}$ and the reorganization energy $\lambda$. While $\mathrm{H}-$ bond donor systems increase the electron transfer rate of quinone systems by making $\Delta \mathrm{G}$ more negative, they also affect the reorganization energy. ${ }^{29}$ A number of articles dealing with this topic suggest that HB donors activate the oxidizing ability of the quinones by stabilizing the radical anion quinone formed subsequent to the electron transfer. One would expect that an anion would participate in a stronger HB than its neutral counterpart. But if that was the only effect, there should not be a large difference between electron-rich and poor quinones, since both of their anion radicals can form this strong H-bond. Another scenario would have HB formation between quinone and HB donor precede the electron transfer. In such 
case, an electron-rich quinone ought to form a stronger HB. In fact, the electrochemical studies of Nocera and Jacobesen indicated that one HB donor molecule binds to the neutral quinone which is then followed by electron transfer. Finally, the radical anion is additionally stabilized by a second donor molecule. ${ }^{20}$

At this juncture, it remains a bit of a puzzle as to why electron-rich and deficient quinones act differently towards H-bond activation. There is little known about the details or even the fundamental nature of the interaction between a proton donor molecule and quinones, either before or after the electron transfer. There are several important question which await an answer. If the interaction of the proton donor with the quinone precedes electron transfer, what are the geometries, energetics and electronic properties of the complexes? Is a H-bonded geometry indeed the preferred structure, or might another type of interaction be favored? It is also important to consider how these issues are affected by the protondonating power of the partner molecule. How does each type of interaction affect the quinone's reduction potential? These same issues must be addressed for the interaction following the addition of an electron. And with respect to trends, why do electron-rich and poor quinones exhibit qualitatively different behavior?

This article reports attempts to answer these questions at the molecular level using quantum mechanical methods. A set of different proton donors is each paired with a range of quinones from very electron-rich to highly deficient. The most stable geometries are ascertained, both before and after an electron is added to the quinone, and the fundamental nature of each interaction is analyzed. The results enable a distinction to be made between electron-rich and poor quinones that is reflective of the experimental results, both in vacuo and in solution.

\section{Computational Details}

A series of o-quinones was considered as indicated in the top portion of Scheme 1. Either two or four substituents $\mathrm{X}$ were added to the quinone in the indicated positions. These substituents included the set $\mathrm{NH}_{2}, \mathrm{Me}, \mathrm{Cl}$, and $\mathrm{F}$. The five proton donors considered here are illustrated in the lower half of Scheme I. Dimethylamine (DMA) is the weakest donor examined, and the two alcohols are a bit stronger. Dimethylurea (DMU) is a strong donor, which includes the possibility of engaging in two HBs simultaneously. Strongest of all is the cationic $\mathrm{CNH}_{2}\left(\mathrm{NHCH}_{3}\right)_{2}{ }^{+}$. 


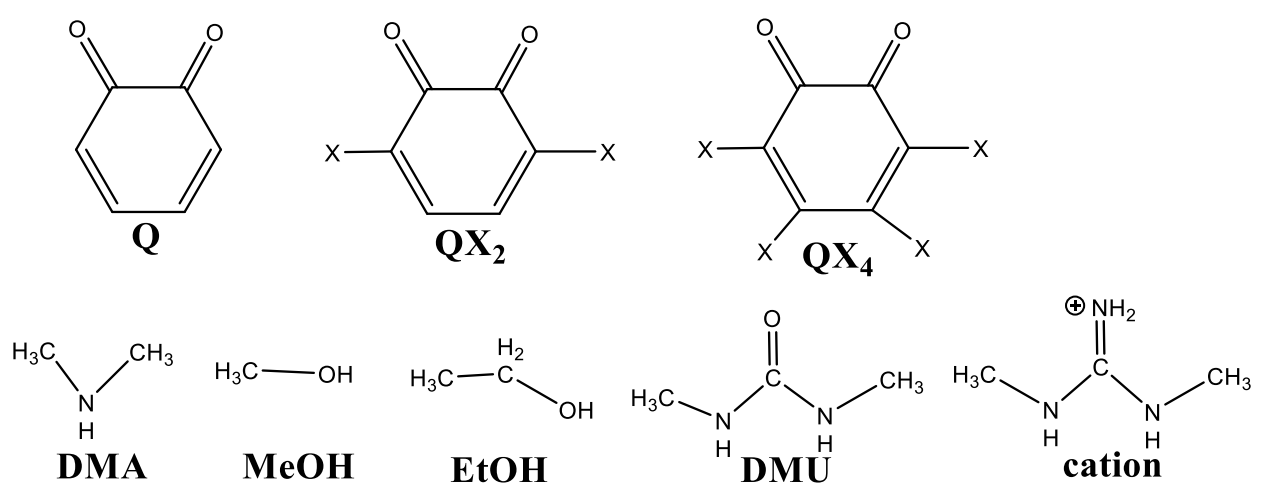

Scheme 1. Quinone and proton donor systems studied

Each of the quinone molecules was paired with a donor system and all the possible minima were identified on the potential energy surface. To ensure each structure represents a true minimum, only geometries with all positive frequencies were taken into account. Density functional theory with M06-2X functional ${ }^{30}$ and aug-cc-pVDZ basis set was applied using Gaussian-09 software. ${ }^{31}$ A good deal of recent work has supported the ability of this level of theory to treat stacked structures with some accuracy as well as H-bonds. ${ }^{32-35}$ Calculations were carried out in the gas phase and in aqueous solvent using the CPCM method. ${ }^{36}$ Charge transfers from one monomer to the other, and their energetic effects, were studied by the Natural Bond Orbital (NBO) method. ${ }^{37}$ The binding energy of each complexes was calculated as the difference between the energy of the complex and the energy sum of the two monomers in their optimized geometries. Each binding energy was corrected for basis set superposition error using the counterpoise method. ${ }^{38}$ The binding energies were further dissected into their constituent components using Symmetry Adapted Perturbation Theory (SAPT) ${ }^{39}$ implemented in the MOLPRO software package. ${ }^{40}$ Atoms-inMolecules(AIM) ${ }^{41}$ calculations were performed by the AIM ALL program. ${ }^{42}$ The electron affinity of each quinone and its various complexes was determined in both vertical and adiabatic schemes. Deprotonation energies were evaluated as the difference in energy between each species, and the entity resulting from removal of the proton of interest.

\section{Result and Discussion}

\section{$\underline{\text { Monomers }}$}

As a first issue, we consider the ease of reduction of the various quinone species. One measure of this property is its electron affinity, eA. The energy released upon acquiring an electron which converts each quinone to its semiquinone radical anion is reported in the first two columns of Table 1. The vertical eA was obtained by adding the electron without allowing the geometry to relax, while the adiabatic analogue 
permitted full geometry optimization of the ensuing anion. The various quinones have been listed in order of greater electron affinity. This order varies from the most electron-donating substituents such as $\mathrm{NH}_{2}$ at the top, down to the electron-withdrawing halogens which have the strongest tendency to attract an excess electron. It is perhaps notable that the F substituent is somewhat less effective than is $\mathrm{Cl}$, as may be seen by comparison of the last two rows. The last column of Table 1 displays the energy of the LUMO of the neutral quinone, into which the electron is to be deposited. The electron-withdrawing power of the substituents at the bottom of the table is verified by the stabilization of this molecular orbital. In summary, all three quantities in Table 1 agree on the order of reduction potential of the various quinones.

The various proton donor species have varying degrees of ability to engage in a HB with the quinones. The most obvious measure of their acidity in this context is their calculated deprotonation energy, reported in Table 2. As expected the amine's NH group requires the most energy to remove its proton, i.e. is the weakest acid, and DMU is the strongest acid. The two alcohols are intermediate between these extremes, with EtOH slightly stronger. The cationic donor of course requires the least energy to remove a proton.

Table 1. Vertical and adiabatic electron affinity of the various quinone monomers, and the energy of its LUMO (kcal/mol)

\begin{tabular}{|c|c|c|c|}
\hline quinone & Vertical & Adiabatic & $\varepsilon(\mathrm{LUMO})$ \\
\hline $\mathrm{Q}\left(\mathrm{NH}_{2}\right)_{4}$ & -27.60 & -37.00 & -46.43 \\
\hline $\mathrm{QMe}_{4}$ & -34.82 & -41.07 & -53.21 \\
\hline $\mathrm{QMe}_{2}$ & -39.09 & -45.48 & -59.49 \\
\hline $\mathrm{Q}$ & -42.77 & -48.81 & -65.66 \\
\hline $\mathrm{QCl}_{2}$ & -56.83 & -63.25 & -76.96 \\
\hline $\mathrm{QF}_{4}$ & -57.32 & -65.61 & -80.85 \\
\hline $\mathrm{QCl}_{4}$ & -63.94 & -70.10 & -81.85 \\
\hline
\end{tabular}

Table 2. Deprotonation energies $(\mathrm{kcal} / \mathrm{mol})$ of proton donor species

\begin{tabular}{|l|l|}
\hline $\mathrm{Me}_{2} \mathrm{NH}$ & 402.95 \\
\hline $\mathrm{MeOH}$ & 389.77 \\
\hline EtOH & 386.97 \\
\hline DMU & 369.15 \\
\hline cation & 253.16 \\
\hline
\end{tabular}

\section{$\underline{\text { Geometries and Energetics of Complexes }}$}

The quinones form two sorts of complexes with the various neutral proton donors. The first category is characterized by $\mathrm{H}$-bonded structures that take advantage of the two $\mathrm{O}$ atoms as proton acceptors. Examples of this sort of structure are provided in Fig 1 for the dimethylquinones. A second type of 
heterodimer displayed in Fig 2 is a stacked structure wherein the partner molecule lies above the plane of the quinone ring. (The coordinates of these geometries are contained in the Supporting Information.) As described in greater detail below, these geometries owe their stability in part to charge transfer from the lone pair of an electronegative atom $(\mathrm{O}$ or $\mathrm{N})$ to the $\pi^{*}$ antibonding orbitals of the quinone $\mathrm{C}=\mathrm{O}$ bonds. The latter stacked complex is the more stable of the two, with the H-bonded geometries serving as secondary minima.

The BSSE-corrected binding energies of both stacked and H-bonded complexes of each of the quinones with the various H-bond donors are reported in Table 3. It is important to note that the cationic donors do not engage in stacked dimers, presumably due to the strength of these charge-amplified H-bonds. The ionic dimers are much more strongly bound, between 18.8 and $34.8 \mathrm{kcal} / \mathrm{mol}$. The binding energies of the neutral HB complexes range between 3.9 and $9.4 \mathrm{kcal} / \mathrm{mol}$, with DMU engaging in the strongest complexes. It might be worthwhile to stress that the greater binding energy of DMU, in comparison to the other neutral donors, is explained in part by its two NH groups, both of which participate in HBs with the quinone $\mathrm{O}$ atoms.

In most cases, the strength of the HB follows the anticipated pattern that electron-withdrawing agents such as the halogens weaken the proton-accepting ability of the quinone $\mathrm{O}$ atoms. The dimethylamine $\mathrm{HB}$ complexes do not obey this trend precisely: for example the electron-poor $\mathrm{QCl}_{2}$ and $\mathrm{QCl}_{4}$ form a stronger $\mathrm{HB}$ dimer than does the electron-rich $\mathrm{QMe}_{4}$, albeit by only a small amount. These deviations are a result of the structures of these particular dimers wherein the amine lies above the quinone plane and the $\mathrm{NH} \cdot \mathrm{O} \mathrm{HBs}$ are supplemented by a certain degree of $\mathrm{NH} \cdot \pi \mathrm{H}$-bonding, as well as some charge transfer from $\sigma(\mathrm{CH})$ the amine to $\pi^{*}(\mathrm{C}=\mathrm{O})$. This auxiliary bonding also accounts for the greater binding energy of the amine than the alcohols which contain a more potent $\mathrm{OH}$ proton donor group.

The HB structures contain a strong element of $n \rightarrow \sigma^{*}$ charge transfer, as is typical of H-bonds. These quantities, reported in Table 4, reinforce the expected trends. The weakest HBs are formed by the amine $\mathrm{NH}$ as compared to the $\mathrm{OH}$ of the alcohols. The larger quantities for DMU arise due to the formation of multiple $\mathrm{NH} \cdot \mathrm{O} \mathrm{HBs}$, and the much higher transfer in the cation donor is typical of ionic HBs. Even more than the total binding energies, the NBO charge transfers obey the trend of diminishing as the quinone electron donor becomes progressively electron poorer, from top to bottom in the table. 

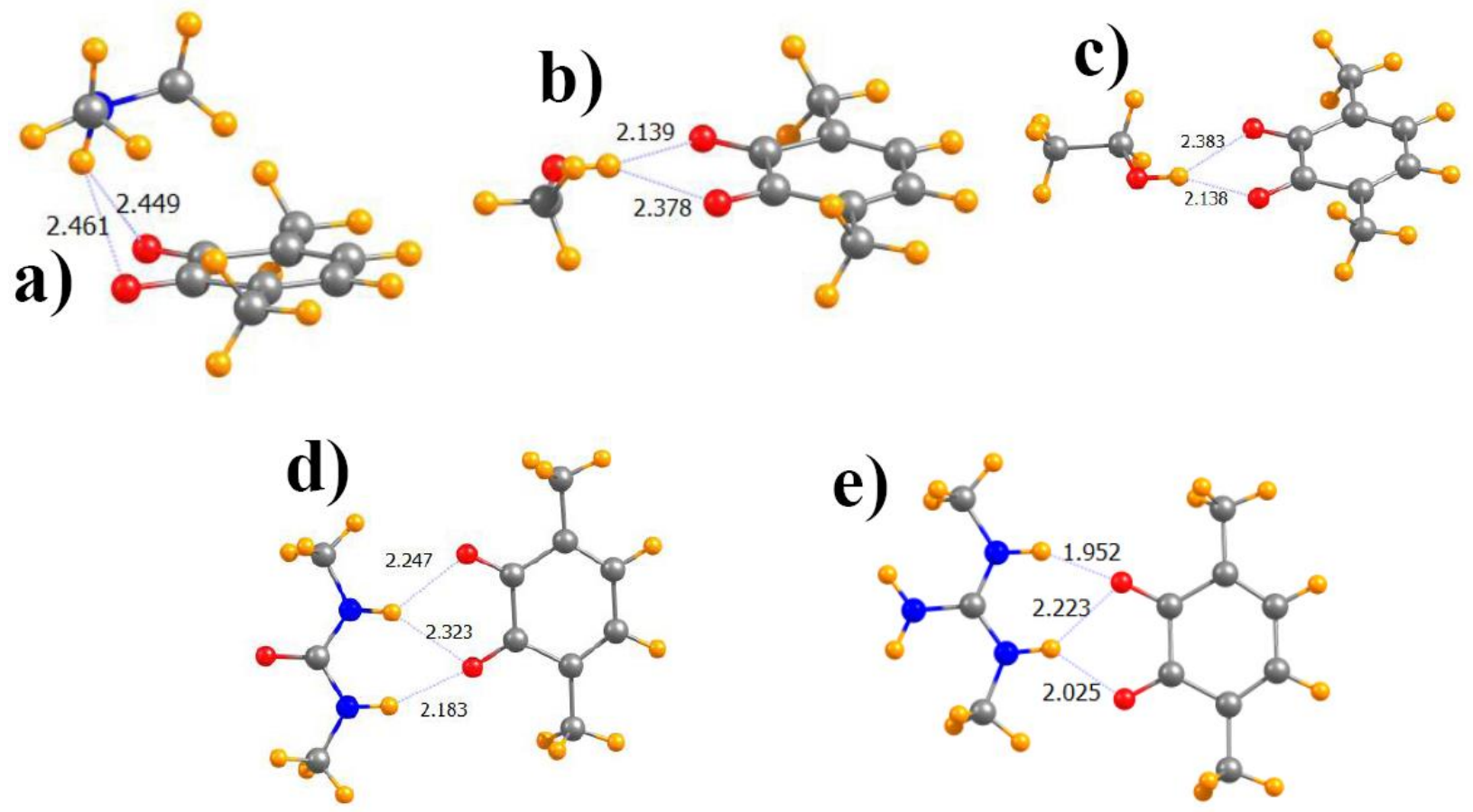

Fig 1. H-bonded geometries of complexes formed by dimethylquinone with proton donors a) $\left.\left(\mathrm{CH}_{3}\right)_{2} \mathrm{~N}, \mathrm{~b}\right)$ $\mathrm{MeOH}$, c) EtOH, d) dimethylurea, e) $\mathrm{CNH}_{2}\left(\mathrm{NHCH}_{3}\right)_{2}{ }^{+}$cation. Distances in $\AA$.
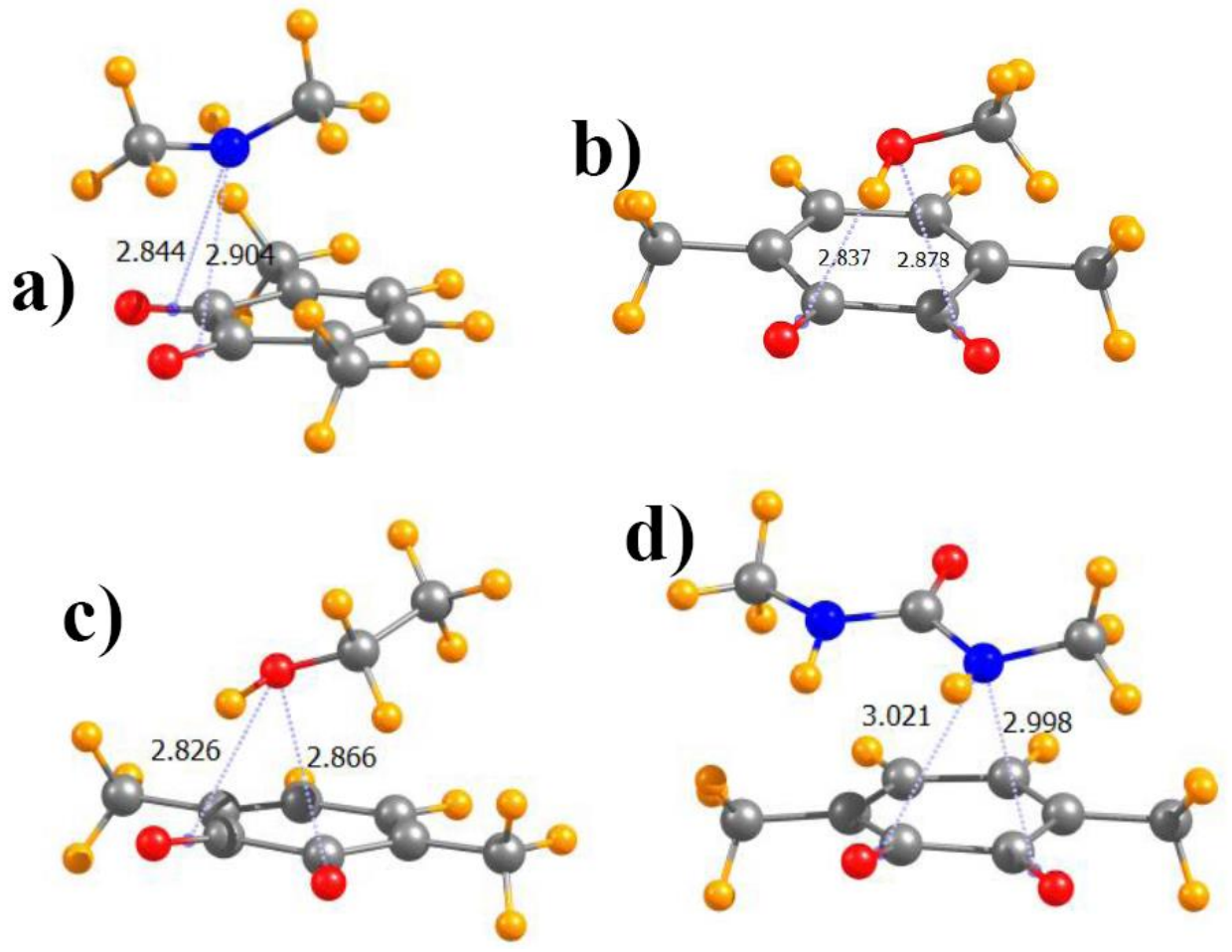

Fig 2. Stacked geometries of complexes formed by dimethylquinone with proton donors a) $\left.\left(\mathrm{CH}_{3}\right)_{2} \mathrm{~N}, \mathrm{~b}\right)$ $\mathrm{MeOH}, \mathrm{c}) \mathrm{EtOH}, \mathrm{d}$ ) dimethylurea. Distances in $\AA$. 
Table 3. Binding energies ( $\mathrm{kcal} / \mathrm{mol})$ of quinones with various $\mathrm{H}$-bond donors

\begin{tabular}{|l|l|l|l|l|l|l|l|l|l|}
\hline \multirow{2}{*}{ Quinone } & \multicolumn{3}{l}{$(\mathrm{Me})_{2} \mathrm{NH}$} & $\mathrm{MeOH}$ & \multicolumn{2}{l|}{ EtOH } & \multicolumn{2}{l|}{ DMU } & $\mathrm{CNH}_{2}\left(\mathrm{NHCH}_{3}\right)_{2}{ }^{+}$ \\
\cline { 2 - 12 } & stacked & $\mathrm{HB}^{\mathrm{a}}$ & stacked & HB & stacked & HB & stacked & $\mathrm{HB}$ & $\mathrm{HB}$ \\
\hline $\mathrm{Q}\left(\mathrm{NH}_{2}\right)_{4}$ & 8.87 & 6.01 & 8.02 & 6.68 & 6.58 & 6.49 & 11.19 & 9.37 & 34.84 \\
\hline $\mathrm{QMe}_{4}$ & 9.18 & 6.34 & 6.85 & 6.43 & 7.74 & 6.28 & 12.82 & 8.84 & 30.46 \\
\hline $\mathrm{QMe}_{2}$ & 9.92 & 6.15 & 6.86 & 5.94 & 7.58 & 5.79 & 10.79 & 8.13 & 27.30 \\
\hline $\mathrm{Q}$ & 10.21 & 5.72 & 6.87 & 5.58 & 7.03 & 5.44 & 10.73 & 7.69 & 26.27 \\
\hline $\mathrm{QCl}_{2}$ & 12.96 & 5.98 & 8.25 & 4.62 & 8.54 & 4.50 & 13.00 & 6.43 & 22.55 \\
\hline $\mathrm{QF}_{4}$ & 12.96 & 5.40 & 8.66 & 4.05 & 8.93 & 3.92 & 12.63 & 5.48 & 18.81 \\
\hline $\mathrm{QCl}_{4}$ & 13.72 & 5.90 & 8.51 & 4.46 & 9.00 & 4.33 & 13.35 & 6.01 & 21.55 \\
\hline
\end{tabular}

anot purely $\mathrm{NH} \cdots \mathrm{O}$ but the combination of $\mathrm{NH} \cdots \mathrm{O}, \mathrm{NH} \cdots \pi$ and $\sigma(\mathrm{CH}) \rightarrow \pi(\mathrm{C}=\mathrm{O})$

As mentioned above the HB minima are secondary to the stacked geometries which form more tightly bound complexes (for the neutral donors). This greater stability margin is as small as $0.4 \mathrm{kcal} / \mathrm{mol}$ for the $\mathrm{MeOH} \cdot \mathrm{QMe}_{4}$ dimer but can be as large as $7.8 \mathrm{kcal} / \mathrm{mol}$ for the dimer pairing $(\mathrm{Me})_{2} \mathrm{NH}$ with $\mathrm{QCl}_{4}$. The stacked geometries also contain a heavy element of charge transfer. In the case of dimethylamine and DMU, transfer from the $\mathrm{N}$ lone pair to the $\pi^{*}(\mathrm{CO})$ antibonding orbitals of the quinone make up the bulk of this quantity, leading to their characterization as lone pair/ $\pi$ complexes. A parallel transfer replaces the $\mathrm{N}$ lone pair by the $\mathrm{O}$ lone pairs for the two alcohols. The energetic magnitude of these charge transfers is displayed in Table 5 for the stacked heterodimers. Just as was noted for the binding energies in Table 3, $(\mathrm{Me})_{2} \mathrm{NH}$ and $\mathrm{DMU}$ whose $\mathrm{N}$ atoms donate charge to the quinone present larger values of $\mathrm{E}(2)$ than do the $\mathrm{O}$ donor alcohols. On the other hand, $\mathrm{E}(2)$ is consistently larger for $(\mathrm{Me})_{2} \mathrm{NH}$ than for DMU, even though their binding energies tend to have the reverse order. The same may be said for $\mathrm{MeOH}$ and $\mathrm{EtOH}$ where the latter is more strongly bound even though its $\mathrm{E}(2)$ is smaller.

Table 4. NBO $\mathrm{O}_{\mathrm{lp}} \rightarrow \sigma(\mathrm{XH})(\mathrm{X}=\mathrm{O}, \mathrm{N})$ charge transfer $\mathrm{E}(2)(\mathrm{kcal} / \mathrm{mol})$ for $\mathrm{HB}$ configurations

\begin{tabular}{|l|c|c|c|c|c|}
\hline & $(\mathrm{Me})_{2} \mathrm{NH}^{\mathrm{a}}$ & $\mathrm{MeOH}$ & $\mathrm{EtOH}$ & $\mathrm{DMU}$ & $\mathrm{CNH}_{2}\left(\mathrm{NHCH}_{3}\right)_{2}{ }^{+}$ \\
\hline $\mathrm{Q}\left(\mathrm{NH}_{2}\right)_{4}$ & 3.19 & 6.09 & 6.36 & 12.67 & 36.92 \\
\hline $\mathrm{QMe}_{4}$ & 3.84 & 6.22 & 6.09 & 11.96 & 28.79 \\
\hline $\mathrm{QMe}_{2}$ & 4.22 & 5.68 & 5.67 & 10.90 & 26.42 \\
\hline $\mathrm{Q}$ & 4.15 & 5.29 & 5.21 & 10.38 & 24.25 \\
\hline $\mathrm{QCl}_{2}$ & 4.16 & 4.62 & 4.57 & 8.98 & 22.28 \\
\hline $\mathrm{QF}_{4}$ & 3.91 & 4.24 & 4.09 & 8.52 & 19.69 \\
\hline $\mathrm{QCl}_{4}$ & 4.11 & 4.55 & 3.35 & 9.02 & 21.94 \\
\hline
\end{tabular}

${ }^{\mathrm{a}}$ contains $\mathrm{O}_{\mathrm{lp}} \rightarrow \sigma(\mathrm{NH}), \pi(\mathrm{CO}) \rightarrow \sigma(\mathrm{NH})$ and also $\sigma(\mathrm{CH}) \rightarrow \pi(\mathrm{CO})$ 
Table 5. NBO charge transfer $\mathrm{E}(2)(\mathrm{kcal} / \mathrm{mol})$ for stacked configurations

\begin{tabular}{|l|c|l|c|l|}
\hline & $(\mathrm{Me})_{2} \mathrm{NH}$ & $\mathrm{MeOH}$ & $\mathrm{EtOH}$ & $\mathrm{DMU}$ \\
\hline $\mathrm{Q}\left(\mathrm{NH}_{2}\right)_{4}$ & 12.80 & 5.69 & 6.18 & 9.35 \\
\hline $\mathrm{QMe}_{4}$ & 11.93 & 7.75 & 6.16 & 6.21 \\
\hline $\mathrm{QMe}_{2}$ & 16.00 & 7.95 & 6.73 & 8.65 \\
\hline $\mathrm{Q}$ & 13.94 & 8.65 & 7.05 & 11.10 \\
\hline $\mathrm{QCl}_{2}$ & 16.80 & 9.61 & 8.62 & 9.28 \\
\hline $\mathrm{QF}_{4}$ & 16.64 & 9.82 & 8.43 & 10.88 \\
\hline $\mathrm{QCl}_{4}$ & 17.10 & 8.16 & 6.89 & 10.42 \\
\hline
\end{tabular}

Whereas NBO would characterize the bonding in the stacked structures as primarily of lone pair/ $\pi$ type based upon the orbitals involved in the primary charge transfer, Atoms-in-Molecules (AIM) analysis of the electron density places a bond path between specific atoms of the two molecules, as is typical of AIM. In the case of the stacked geometry of $\mathrm{MeOH}$ with $\mathrm{Q}$, for example, the bond path leads from the $\mathrm{MeOH} \mathrm{O}$ atom to one of the two $\mathrm{C}$ atoms bound to $\mathrm{O}$.

It is worth stressing an important set of trends in the energetic data in Table 3. As the quinone transitions from electron-rich to poor, i.e. from top to bottom in the table, the HB binding energy tends to diminish. The stacked structures, however, obey an opposite pattern, strengthening as the quinone becomes more electron-deprived. One can understand this behavior on the basis of the charge transfers detailed above. Formation of a HB is weakened as the quinone, and thus its $\mathrm{O}$ atoms, become less negative as a result of electron-withdrawing substituents. The stacked dimers are dependent on transfer in the other direction, to the quinone from the $\mathrm{O}$ or $\mathrm{N}$ lone pairs of the partner molecule. The presence of electronwithdrawing groups such as halogens can thus be expected to boost this transfer and thus raise the binding energy.

Another view of these trends is purely electrostatic in origin. The molecular electrostatic potentials (MEPs) of three of the quinones are displayed in Fig 3 where blue and red colors respectively indicate positive and negative regions. As one transitions from the most electron-releasing $\mathrm{NH}_{2}$ substituents on the left to the most electron-withdrawing $\mathrm{Cl}$ on the right, the red negative regions around the $\mathrm{O}$ atoms diminish in magnitude, which would lead to a reduced H-bonding ability, consistent with the pattern in Table 3. One may note also a small blue positive region above the midpoint of the two $\mathrm{C}$ atoms that are bound to $\mathrm{O}$, an area that might be termed a $\pi$-hole. The intensity of this $\pi$-hole increases as the substituents become more electron-withdrawing. The magnitude of this hole can be measured by the maximum of the MEP, which is displayed by the numerical values in Fig 3, which shows the expected rise as the substituents vary from electron-releasing $\mathrm{NH}_{2}$ to electron-withdrawing $\mathrm{Cl}$. It follows then that the electrostatic attraction of the 
quinone to a $\mathrm{O}$ or $\mathrm{N}$ atom that lies above this $\pi$-hole will likewise be enhanced, accounting for the larger binding energies of the stacked geometries from top to bottom in Table 3.
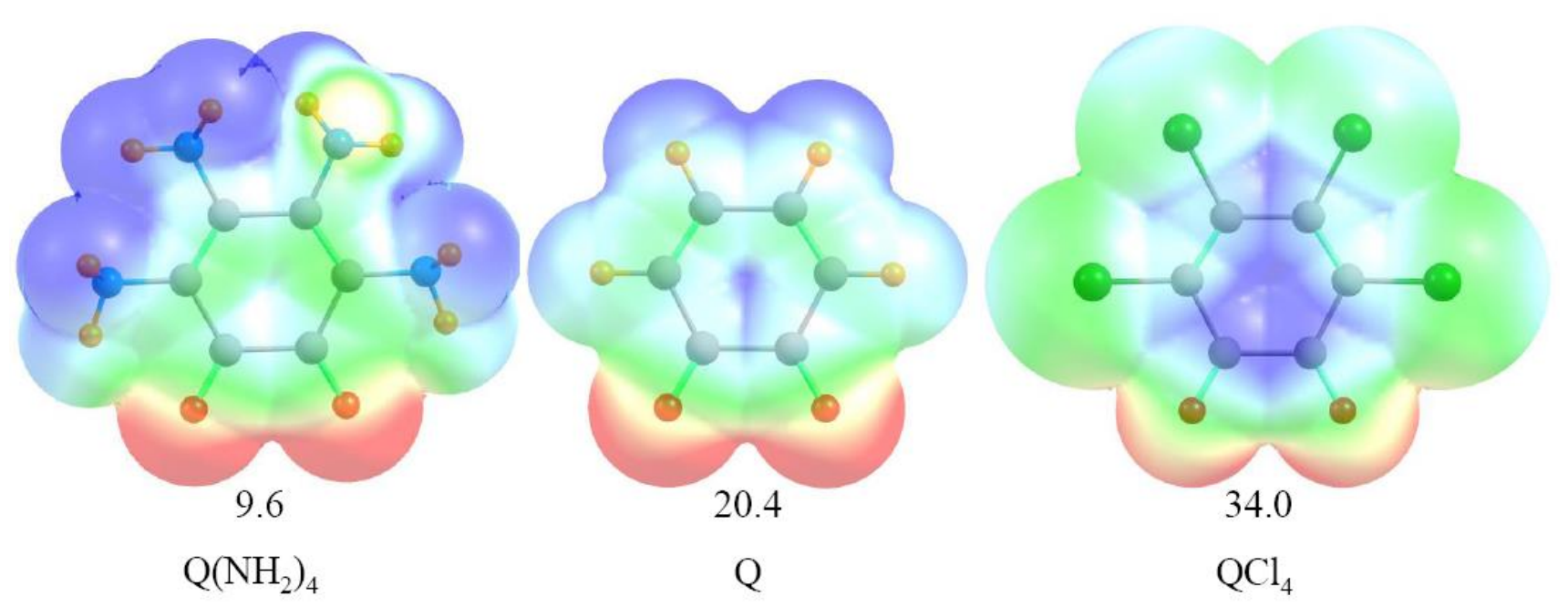

Fig 3. Molecular electrostatic potential (MEP) surrounding each of the indicated quinones on a surface corresponding to $1.5 \mathrm{x}$ van der Waals radius. Blue and red colors indicate maxima and minima, respectively, \pm 0.005 au. Numerical values refer to $\mathrm{V}_{\mathrm{s}, \max }(\mathrm{kcal} / \mathrm{mol})$ at the $\pi$-hole above the C-C bond connecting the two $\mathrm{CO}$ groups, on the $\rho=0.001$ au isodensity surface.

Further insight into the stronger binding of the stacked vs the H-bonded structures can be gleaned from a decomposition of the total binding energies. An SAPT analysis reveals that all aspects of the interaction are enhanced in the stacked geometries. The electrostatic component is magnified by a factor of 1.3-2.6. The enlargements of the dispersion is larger, in the 2.1-3.6 range while induction larger still: 2.4-6.0. The increases in the latter two quantities are consistent with the large induction and dispersion expected for a stacked geometry.

This idea is reinforced by examination of the electronic redistributions caused by formation of the various complexes. Fig 4 was computed by subtracting the electron densities of the two individual monomers from that of the full complex. The purple areas represent regions where density is increased as a result of formation of the dimer, and losses are indicated by green. The system chosen for illustration is the DMU/quinone pair. The H-bonded structure on the left shows the classic HB fingerprint of loss surrounding the bridging $\mathrm{H}$ atoms, and increases in the regions of the proton-accepting $\mathrm{O}$ lone pairs of quinone. The pattern of the stacked structure on the right shows larger contours and thus greater charge shifts. These shifts are also more delocalized involving larger portions of each molecule, consistent with the larger induction energy revealed by SAPT. In more detail, there is substantial charge gain occurring 
both above and below the quinone $\mathrm{O}$ atoms, and losses on the attached $\mathrm{C}$ atoms. In the context of DMU, The $\mathrm{H}$ atoms suffer some loss, while there appears to be a certain degree of shift from the $\sigma$ to the $\pi$-system in the vicinity of the two $\mathrm{N}$ atoms.

NMR chemical shifts of protons are a common indicator of the presence and strength of a HB. But they can also provide information about some of the fundamental characteristics of other types of interactions. The shifts of the H-bonding protons are reported in Table $\mathrm{S} 1$ relative to the uncomplexed monomer. As expected these protons suffer a loss of shielding, i.e. downfield shift, for each of the H-bonding conformations. Secondly, the shifts are larger for the more strongly H-bonding quinones at the top of Table $\mathrm{S} 1$ in the Supporting Information, and largest for the cationic proton donor that engages in the strongest HBs. For the stacked structures, on the other hand, the same protons are more strongly shielded in the complex than in the monomer, albeit by less than $1 \mathrm{ppm}$. The density difference map, with its yellow density loss contours around these protons, might have argued for a lower shielding. However, the observed increased shielding may be due to the ring currents within the conjugated quinone system, much as phenyl rings are known to increase the shielding of atoms placed above them.

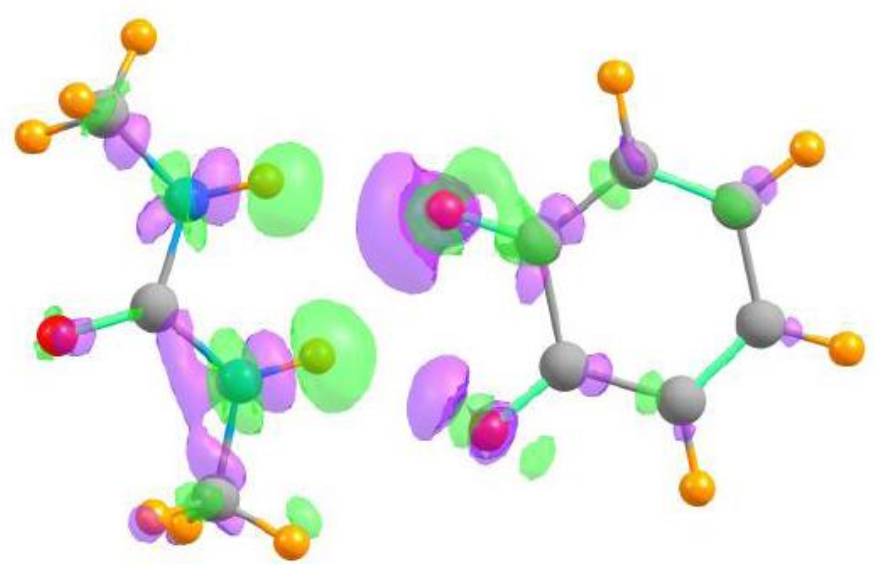

a) H-bonded

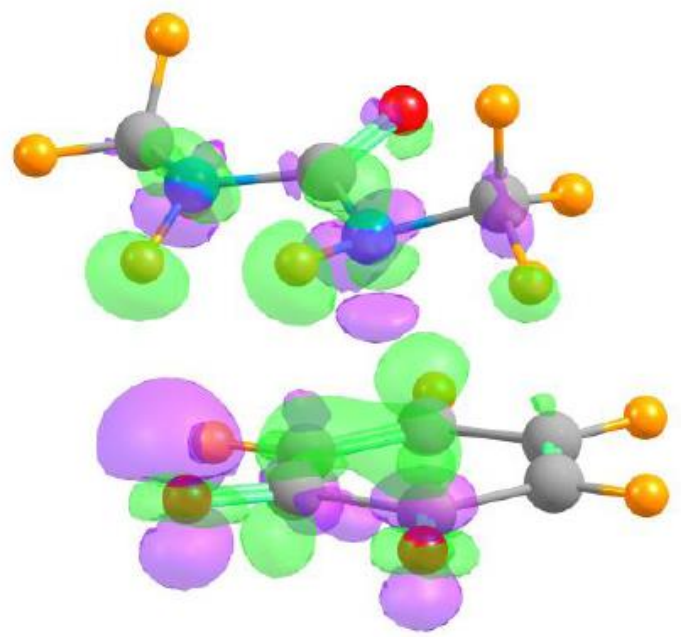

b) stacked

Fig 4. Electron density difference map of a) H-bonded and b) stacked structures of quinone with dimethylurea. Purple regions indicate increased density resulting from formation of complex; losses are shown in green. Contours represent \pm 0.001 au.

It might be added finally, that the lone pair $\rightarrow \pi *$ transfers that characterize the stacked structures is not particular to 1,2 benzoquinone. Parallel calculations with the 1,4 benzoquinones led to similar results, with stacked dimers preferred over HB structures.

$\underline{\text { Radical Semiquinone Anion Complexes }}$ 
After accepting an electron the quinone transitions to a radical anion semiquinone state. The global minimum for the complexes involving the radicals are of $\mathrm{H}$-bonding type, with binding energies displayed in Table 6. The stacked structures common to the neutral quinones do not represent minima on the surface of the semiquinone radicals. Representative structures of the dimethyl semiquinone are illustrated in Fig 5. Comparison with the HB geometries in Fig 1 indicates little fundamental differences, other than a contraction of the intermolecular distances.

The presence of a full charge on one of the subunits is expected to amplify various facets of the intermolecular interaction. And indeed the binding energies in Table 6 are considerably larger than for the neutral HB structures in Table 3. The charge magnification effect is smallest for the amine (3.5 - 6.3 $\mathrm{kcal} / \mathrm{mol}$ ) and largest for DMU with increases between 15.4 and $18.8 \mathrm{kcal} / \mathrm{mol}$. Even more impressive is the increment of $76-83 \mathrm{kcal} / \mathrm{mol}$ for the cationic proton donor, with binding energies in excess of 100 $\mathrm{kcal} / \mathrm{mol}$. In terms of relative growth, the placement of a negative charge on the semiquinone roughly doubles the HB interaction energy of the amine, and magnifies this quantity for the alcohols, DMU, and the cation by respective factors of 2-3, 3-4; and 3-5. Like the neutral systems, the anionic semiquinone HB energies obey the trend amine < alcohol $<$ DMU < cation, although the two alcohols reverse with one another. The expected trend of a weakening HB as one moves down a column of Table 6, from electronrich to electron-deficient semiquinone proton acceptor is not strictly adhered to.
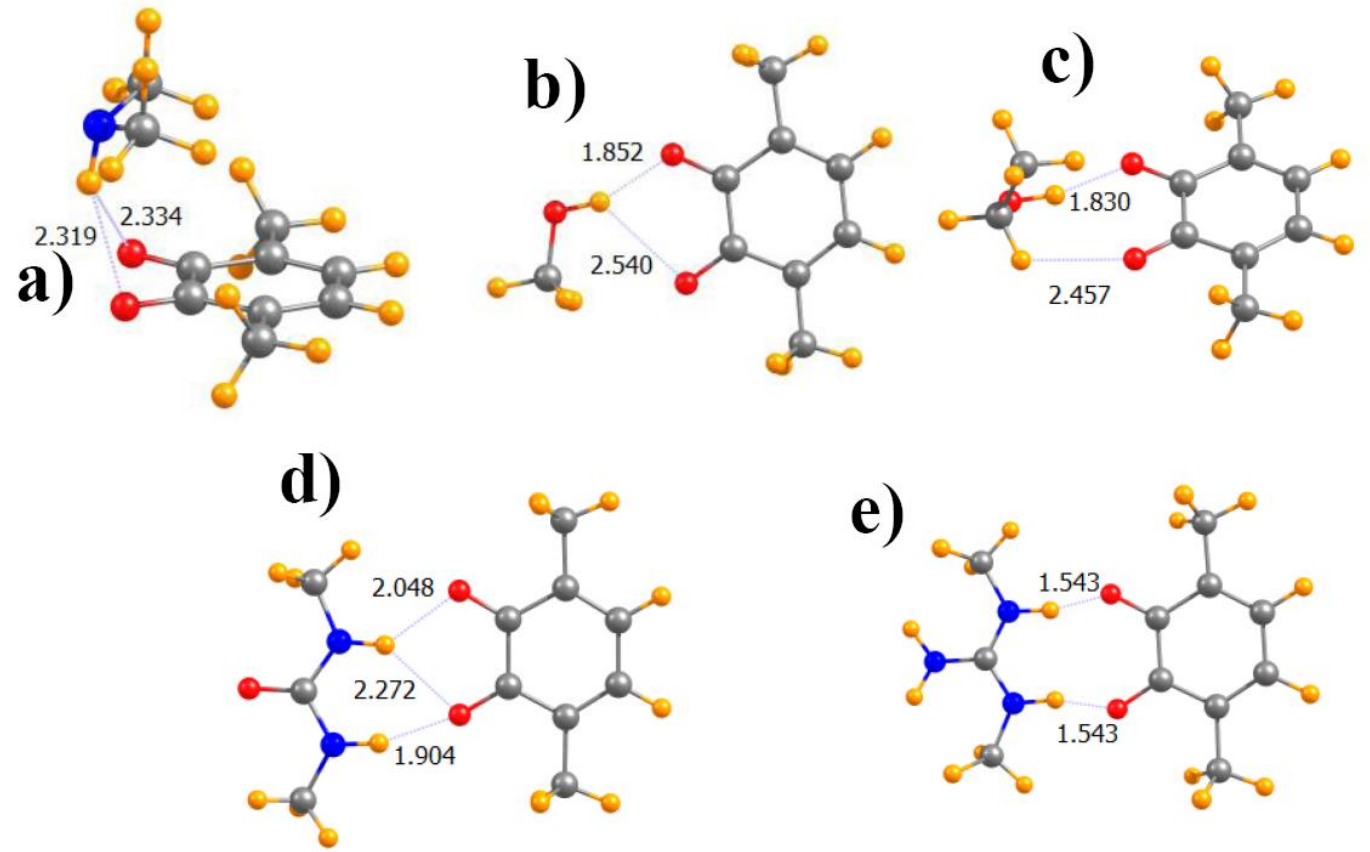

e)

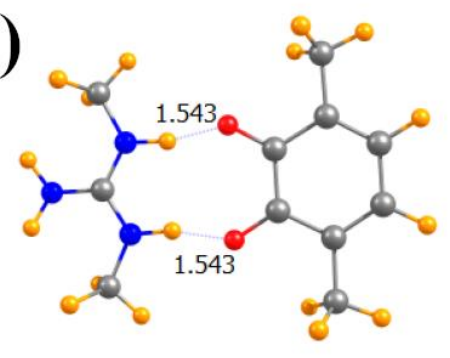

Fig 5. Geometries of complexes formed by dimethylquinone anion radical with proton donors a) $\left.\left(\mathrm{CH}_{3}\right)_{2} \mathrm{~N}, \mathrm{~b}\right)$ $\mathrm{MeOH}$, c) EtOH, d) dimethylurea, e) $\mathrm{CNH}_{2}\left(\mathrm{NHCH}_{3}\right)_{2}{ }^{+}$cation. Distances in $\AA$. 
Table 6. Binding energies $(\mathrm{kcal} / \mathrm{mol})$ of radical semiquinone anions with various $\mathrm{H}$-bond donors

\begin{tabular}{|l|l|l|l|l|l|}
\hline & $(\mathrm{Me})_{2} \mathrm{NH}$ & $\mathrm{MeOH}$ & $\mathrm{EtOH}$ & $\mathrm{DMU}$ & $\mathrm{CNH}_{2}\left(\mathrm{NHCH}_{3}\right)_{2}{ }^{+}$ \\
\hline $\mathrm{Q}\left(\mathrm{NH}_{2}\right)_{4}{ }^{-}$ & 10.37 & 13.99 & 14.12 & 24.75 & 110.22 \\
\hline $\mathrm{QMe}_{4}{ }^{-}$ & 12.32 & 15.95 & 16.96 & 26.83 & 111.83 \\
\hline $\mathrm{QMe}_{2}{ }^{-}$ & 12.32 & 15.62 & 16.68 & 26.52 & 110.51 \\
\hline $\mathrm{Q}^{-}$ & 11.68 & 15.56 & 16.61 & 26.48 & 111.87 \\
\hline $\mathrm{QCl}_{2} \cdot \cdot$ & 10.51 & 13.44 & 14.19 & 23.10 & 102.99 \\
\hline $\mathrm{QF}_{4}^{-} \cdot$ & 10.06 & 13.36 & 14.16 & 22.84 & 101.33 \\
\hline $\mathrm{QCl}_{4}{ }^{-}$ & 9.81 & 12.53 & 13.20 & 21.43 & 97.98 \\
\hline
\end{tabular}

As would be anticipated for the stronger HBs involving the anion, the NBO charge transfers in Table $\mathrm{S} 2$ are similarly enlarged when compared to their neutral analogues in Table 4. One again sees the similar trend of a general weakening as the semiquinone substituent becomes more electron-withdrawing. Also commensurate with the neutral systems, DMU shows the largest charge transfer and $(\mathrm{Me}){ }_{2} \mathrm{NH}$ the least. Effect of Complexation upon Reduction

A central issue motivating this work is an elucidation of how the formation of a complex affects the reduction process of each quinone. In other words, does the complexation raise or lower the electron affinity of the quinone. The change in the electron affinity can be equated by simple Hess's Law considerations with the difference between the binding energy of the quinone as compared to the corresponding anionic radical semiquinone. That is, the increase in the electron affinity caused by the formation of the complex is equal to the increase in the binding energy caused by adding an electron to the quinone:

$$
\mathrm{eA}(\mathrm{PD}-\mathrm{Q})-\mathrm{eA}(\mathrm{Q})=\mathrm{E}_{\mathrm{b}}\left(\mathrm{Q}^{-}\right)-\mathrm{E}_{\mathrm{b}}(\mathrm{Q})
$$

where PD-Q refers to the complex and $\mathrm{E}_{\mathrm{b}}$ corresponds to the binding energy of the indicated species with PD.

The quantities in Eq (1) were computed by comparing the binding energies of the anionic radical semiquinones in Table 6 with the comparable quantities in Table 3 for the neutral quinones. (It should be noted that the more stable of the latter dimers were the stacked structures, not the H-bonded geometries.) The increment of the electron affinity of each quinone associated with its association with the various proton donor molecules is reported in Table S3, and the data depicted graphically in Fig 6.

Focusing first on the neutral proton donors in the lower part of Fig 6, these increments are all below $16 \mathrm{kcal} / \mathrm{mol}$. There is a clear trend in that the strongest proton donor, DMU, causes the largest enhancement, and the weakest amine the smallest; the two alcohols are intermediate between these two extremes. There is another pattern present, regardless of the identity of the proton donor. The electron 
affinity enhancement is largest for the four quinone species on the left, and smallest for those on the right. That is, the electron-rich quinones undergo a larger increase in their electron affinity upon association with a proton-donor molecule than do the electron-deficient species with halogen substituents. In a quantitative sense, this difference between electron-rich and poor quinones is roughly $5 \mathrm{kcal} / \mathrm{mol}$.

It is interesting that there is little difference between the four electron-rich, nor amongst the three electron-poor quinones. It is also intriguing to observe negative quantities when the $\mathrm{Me}_{2} \mathrm{NH}$ associates with the three most electron-poor quinones. This result is due to the poor proton-donating ability of this amine. Its H-bonding energy with even the anionic semiquinone $(9-10 \mathrm{kcal} / \mathrm{mol})$ is smaller than the strong association energy of the amine in its stacked arrangement with the corresponding neutral quinones (13-14 $\mathrm{kcal} / \mathrm{mol})$.

The patterns for the cationic donor in the upper part of Fig 6 are a bit different. First of all, the cationic species induces a much larger increment in the quinone's electron affinity, between 75 and $85 \mathrm{kcal} / \mathrm{mol}$. Secondly, the principle observed for the neutrals, that the electron-poor quinones undergo a smaller increment than do their electron-rich counterparts, is largely absent. In fact, it is the unsubstituted quinone that shows the largest increment, and the nominally electron-rich tetraamino-substituted analogue the smallest.

The reader should recall that the most stable complex of each of the neutral proton donors with any of the quinones is a stacked geometry. It might be of interest to wonder how the trends in Fig 6 might be affected if the H-bonded geometry were used, not only for the reduced semiquinone, but also for the neutral species. The results in this case are reported in Table S4 and illustrated in Fig S1 where it may be seen first that the electron affinity enhancements are quantitatively a bit larger here than in Fig 6. But perhaps more importantly, there is much less alteration of the data from left to right. That is, if the H-bonded geometry is used for both the neutral quinone and its anionic correlate, there is a much lesser distinction between electron-rich and poor species. (The results for the cationic donor are identical in Figs 6 and S1 because it is the H-bonded species which is the global minimum for the neutral as well as anionic quinone.) 


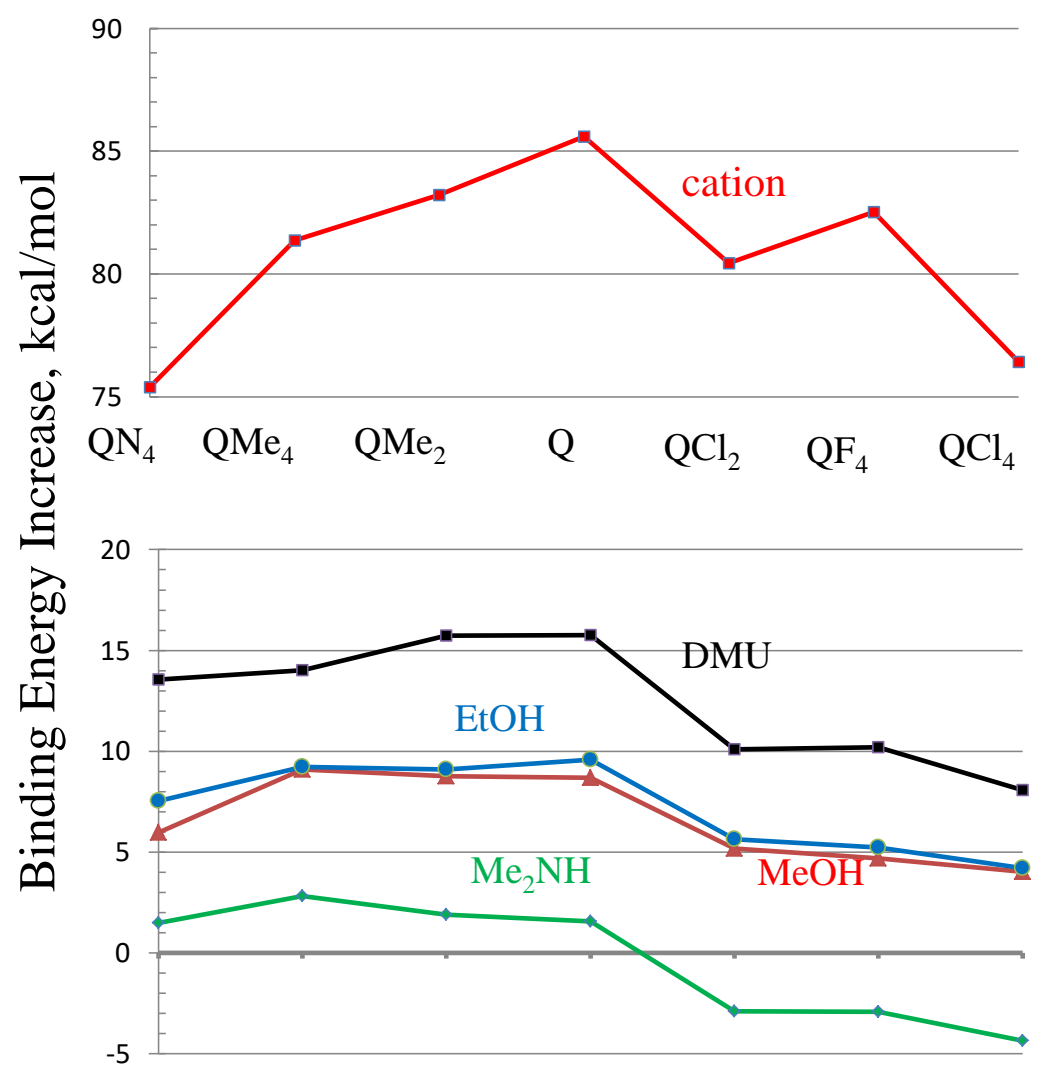

Fig 6. Change in binding energy to proton donor molecule caused by reduction of the quinone to radical anion semiquinone.

One might think there ought to be a connection between the electron affinity of a given species such as a quinone, and the energy of the LUMO into which an added electron would find itself. For example, a lowering of the LUMO energy $\varepsilon$ should make the species more attractive to an incoming electron, raising its electron affinity. However, the opposite was noted in the stacked, most stable, geometries of the various quinone/proton donor complexes. The stacking caused the energy of the quinone's LUMO to rise, i.e. become less negative. This rise was on the order of 3-16 kcal/mol. This trend can be understood on the basis of the observation that the formation of the stacked dimer is associated with a certain amount of charge transfer from the proton donor molecule into the quinone. This added electron density would make the quinone less attractive to an incoming electron. And in fact, the degree of increase of $\varepsilon$ is roughly proportional to the charge transfers documented in Table 5. In any case, this trend is opposite to the aforementioned energetic pattern of enhanced electron affinity of the complex in comparison to the quinone monomer. One can thus conclude that monitoring of the LUMO energy would lead to an incorrect 
conclusion. It is of interest to note finally that because the formation of a HB results in electron donation from the quinone, the LUMO energy of the quinone drops when this HB is formed.

\section{Solvation Effects}

The methods to this point were designed to get to the most fundamental properties of the molecules involved, free of complicating effects. On the other hand, as the practical applications of these results will generally involve placing the systems within a solvent, it is worthwhile to examine how the principles might be affected by solvation effects. The calculations were repeated by reoptimizing the geometries within the context of aqueous solvation, modeling the effects of hydration by the CPCM approach. The binding energies of the quinones with the various proton donor molecules are reported in Table S5. As expected the aqueous environment reduces the various interactions by variable amounts. The binding energies of the amine suffer only a small reduction, on the order of 1 or $2 \mathrm{kcal} / \mathrm{mol}$, with larger decrements for the systems that engage in tighter binding. These reductions tend to be larger for the stacked structures than for the H-bonded geometries. On a percentage basis, the decreases are typically on the order of roughly $15-30 \%$, but larger for the cationic donor, on the order of $70 \%$. The effects of solvation upon the binding energies of the semiquinones are apparent in a comparison of the data in Tables 6 and S6. One again sees reductions, and of a larger magnitude, roughly $50 \%$ for the neutral proton donors, and as much as $85 \%$ for the cation.

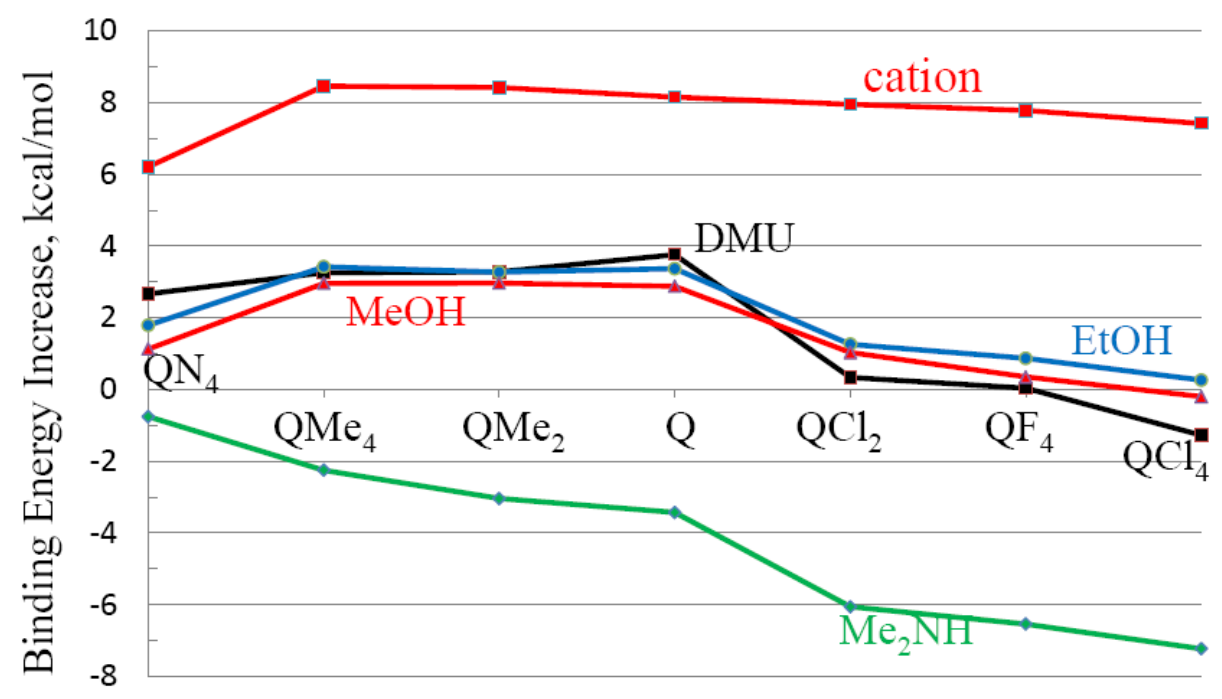

Fig 7. Change in binding energy to proton donor molecule caused by reduction of the quinone to radical anion semiquinone in aqueous solvent. 
When all of these solvent effects are considered in terms of the increase of quinone electron affinity caused by complexation, the graphical form of the data is seen in Fig 7. Comparison with the unsolvated data in Fig 6 reveals a reduction in magnitude of the effects. For example, the gas-phase affinity enhancements were as large as $16 \mathrm{kcal} / \mathrm{mol}$ for neutral donors, and up to $85 \mathrm{kcal} / \mathrm{mol}$ for the cation. The respective solvated maxima are 4 and $9 \mathrm{kcal} / \mathrm{mol}$. But perhaps most importantly, the patterns are changed only very little. Whether gas-phase or solvated, the electron-rich quinones on the left show the largest change, and the electron-poor quinones the smallest, at least for the neutral donors.

\section{Conclusions}

In summary, the neutral proton donors prefer a stacked geometry over a HB structure with the various quinones. N-containing amine and urea derivative form stronger stacked $n \rightarrow \pi^{*}$ complexes with the quinones than do alcohols. Electron-poor quinones, e.g. with halogen substituents, are more strongly bound than are electron-rich quinones, consistent with the idea that electron density is being transferred to the quinone. A cationic proton donor, on the other hand, forms only a H-bonded complex. Following the reduction of the quinone to a radical anion semiquinone, complexation with each proton donor leads to a HB structure, much more strongly bound than the pre-reduced complex. For example, the binding energy with the cationic donor exceeds $100 \mathrm{kcal} / \mathrm{mol}$.

Comparison of the binding energies of the neutral and anionic quinones leads to evaluation of the increase in electron affinity of the quinone associated with its association with each proton donor. This quantity obeys the trend amine < alcohol < urea < cation. The electron affinity increase is as much as 15 $\mathrm{kcal} / \mathrm{mol}$ for the neutral proton donors, and as high as $85 \mathrm{kcal} / \mathrm{mol}$ for the cation. Most importantly, the increased tendency toward reduction caused by the addition of the proton donor molecule is largest for the electron-rich quinones and smallest for the electron-poor species. These same patterns are in evidence when the systems are immersed in aqueous solvent, although the numerical values are smaller. Unlike the other species, the association of the amine induces a reduction in the quinone's electron affinity, albeit only in water. It is reasonable to suppose that the effects of a less polar solvent than water would lead to results intermediate between these two extremes, but still obeying the same patterns.

Turek et $\mathrm{al}^{20}$ had recently observed that the electron deficient chloranil, corresponding to our $\mathrm{QCl}_{4}$, could be activated as an oxidizing agent via addition of a H-bonding agent. This result is consistent with our own finding that the electron affinity of $\mathrm{QCl}_{4}$ is raised when proton donors such as alcohols or DMU are added, and by much more so when the donor carries a positive charge. It is anticipated that the incorporation of a dicationic species, as examined by Turek et al, into the calculations would cause an even larger enhancement, consistent with their observations. 


\section{ACKNOWLEDGMENTS}

Computer, storage and other resources from the Division of Research Computing in the Office of Research and Graduate Studies at Utah State University are gratefully acknowledged.

\section{SUPPORTING INFORMATION}

NMR shifts, NBO perturbation energies, binding energies, and their increments, and atomic coordinates of optimized structures. This material is available free of charge via the Internet at http://pubs.acs.org. 


\section{REFERENCES}

(1) Biggins, J. Progress in Photosynthesis Research: Volume 4 Proceedings of the VIIth International Congress on Photosynthesis Providence, Rhode Island, USA, August 10-15, 1986; Springer Netherlands, 2012.

(2) Ernster, L.; Dallner, G. Biochimica et Biophysica Acta (BBA) - Molecular Basis of Disease 1995, 1271, 195.

(3) Åberg, F.; Appelkvist, E.-L.; Dallner, G.; Ernster, L. Arch. Biochem. Biophys. 1992, 295, 230.

(4) Shrestha, J. P.; Chang, C.-W. T. Bioorg. Med. Chem. Lett. 2013, 23, 5909.

(5) Shrestha, J. P.; Fosso, M. Y.; Bearss, J.; Chang, C.-W. T. Eur. J. Med. Chem. 2014, 77, 96.

(6) Bachur, N. R.; Gordon, S. L.; Gee, M. V. Cancer Research 1978, 38, 1745.

(7) Meazza, G.; Dayan, F. E.; Wedge, D. E. J. Agric. Food. Chem. 2003, 51, 3824.

(8) DuVall, S. H.; McCreery, R. L. J. Am. Chem. Soc. 2000, 122, 6759.

(9) Neumann, R.; Khenkin, A. M.; Vigdergauz, I. Chem. Eur. J. 2000, 6, 875.

(10) Wendlandt, A. E.; Stahl, S. S. J. Am. Chem. Soc. 2014, 136, 11910.

(11) Tse, D. C.-S.; Kuwana, T. Anal. Chem. 1978, 50, 1315.

(12) Caruana, L.; Fochi, M.; Bernardi, L. Molecules 2015, 20, 11733.

(13) Zhang, X.-Z.; Du, J.-Y.; Deng, Y.-H.; Chu, W.-D.; Yan, X.; Yu, K.-Y.; Fan, C.-A. J. Org. Chem. 2016, 81, 2598.

(14) Grennberg, H.; Gogoll, A.; Baeckvall, J. E. J. Org. Chem. 1991, 56, 5808.

(15) Zhu, Z.; Hong, M.; Guo, D.; Shi, J.; Tao, Z.; Chen, J. J. Am. Chem. Soc. 2014, 136, 16461.

(16) Pirnat, K.; Dominko, R.; Cerc-Korosec, R.; Mali, G.; Genorio, B.; Gaberscek, M. J. Power Sources 2012, 199, 308.

(17) Lee, J.; Kim, H.; Park, M. J. Chem. Mater. 2016.

(18) Graige, M. S.; Paddock, M. L.; Bruce, J. M.; Feher, G.; Okamura, M. Y. J. Am. Chem. Soc. 1996, $118,9005$.

(19) Song, N.; Gagliardi, C. J.; Binstead, R. A.; Zhang, M.-T.; Thorp, H.; Meyer, T. J. J. Am. Chem. Soc. 2012, 134, 18538.

(20) Turek, A. K.; Hardee, D. J.; Ullman, A. M.; Nocera, D. G.; Jacobsen, E. N. Angew. Chem. Int. Ed. 2016, 55, 539.

(21) Okamoto, K.; Ohkubo, K.; Kadish, K. M.; Fukuzumi, S. J. Phys. Chem. A 2004, 108, 10405.

(22) Yuasa, J.; Yamada, S.; Fukuzumi, S. Angew. Chem. Int. Ed. 2007, 46, 3553.

(23) Fukuzumi, S.; Kitaguchi, H.; Suenobu, T.; Ogo, S. Chem. Commun. 2002, 1984.

(24) Uno, B.; Okumura, N.; Goto, M.; Kano, K. J. Org. Chem. 2000, 65, 1448.

(25) Staley, P. A.; Lopez, E. M.; Clare, L. A.; Smith, D. K. J. Phys. Chem. C 2015, 119, 20319.

(26) Ge, Y.; Miller, L.; Ouimet, T.; Smith, D. K. J. Org. Chem. 2000, 65, 8831.

(27) Gupta, N.; Linschitz, H. J. Am. Chem. Soc. 1997, 119, 6384.

(28) Marcus, R. A. Angew. Chem. Int. Ed. 1993, 32, 1111.

(29) Yago, T.; Gohdo, M.; Wakasa, M. J. Phys. Chem. B 2010, 114, 2476.

(30) Zhao, Y.; Truhlar, D. G. Theor. Chem. Acc. 2007, 120, 215.

(31) Frisch, M. J.; Trucks, G. W.; Schlegel, H. B.; Scuseria, G. E.; Robb, M. A.; Cheeseman, J. R.; Scalmani, G.; Barone, V.; Mennucci, B.; Petersson, G. A.; Nakatsuji, H.; Caricato, M.; Li, X.; Hratchian, H. P.; Izmaylov, A. F.; Bloino, J.; Zheng, G.; Sonnenberg, J. L.; Hada, M.; Ehara, M.; Toyota, K.; Fukuda, R.; Hasegawa, J.; Ishida, M.; Nakajima, T.; Honda, Y.; Kitao, O.; Nakai, H.; Vreven, T.; Montgomery Jr., J. A.; Peralta, J. E.; Ogliaro, F.; Bearpark, M. J.; Heyd, J.; Brothers, E. N.; Kudin, K. N.; Staroverov, V. N.; Kobayashi, R.; Normand, J.; Raghavachari, K.; Rendell, A. P.; Burant, J. C.; Iyengar, S. S.; Tomasi, J.; Cossi, M.; Rega, N.; Millam, N. J.; Klene, M.; Knox, J. E.; Cross, J. B.; Bakken, V.; Adamo, C.; Jaramillo, J.; Gomperts, R.; Stratmann, R. E.; Yazyev, O.; Austin, A. J.; Cammi, R.; Pomelli, C.; Ochterski, J. W.; Martin, R. L.; Morokuma, K.; Zakrzewski, 
V. G.; Voth, G. A.; Salvador, P.; Dannenberg, J. J.; Dapprich, S.; Daniels, A. D.; Farkas, Ö.;

Foresman, J. B.; Ortiz, J. V.; Cioslowski, J.; Fox, D. J.; Gaussian, Inc.: Wallingford, CT, USA, 2009.

(32) Zhao, Y.; Truhlar, D. G. Acc. Chem. Res. 2008, 41, 157.

(33) Guin, M.; Patwari, G. N.; Karthikeyan, S.; Kim, K. S. PCCP 2011, 13, 5514.

(34) Guin, M.; Patwari, G. N.; Karthikeyan, S.; Kim, K. S. PCCP 2009, 11, 11207.

(35) Momeni, Z.; Ebrahimi, A. Struct. Chem. 2015, 27, 731.

(36) Cossi, M.; Rega, N.; Scalmani, G.; Barone, V. J. Comput. Chem. 2003, 24, 669.

(37) Foster, J. P.; Weinhold, F. J. Am. Chem. Soc. 1980, 102, 7211.

(38) Boys, S. F.; Bernardi, F. Mol. Phys. 1970, 19, 553.

(39) Szalewicz, K. WIREs Comput Mol Sci 2012, 2, 254.

(40) Werner, H.-J.; Knowles, P. J.; Knizia, G.; Manby, F. R.; Schütz, M. WIREs Comput Mol Sci 2012, 2, 242.

(41) Bader, R. F. W. Atoms in Molecules: A Quantum Theory; Clarendon Press, 1994.

(42) Keith, T. A. AIMAll (Version 13.11.04). TK Gristmill Software, Overland Park, KS, USA, 2013. 
TOC graphic

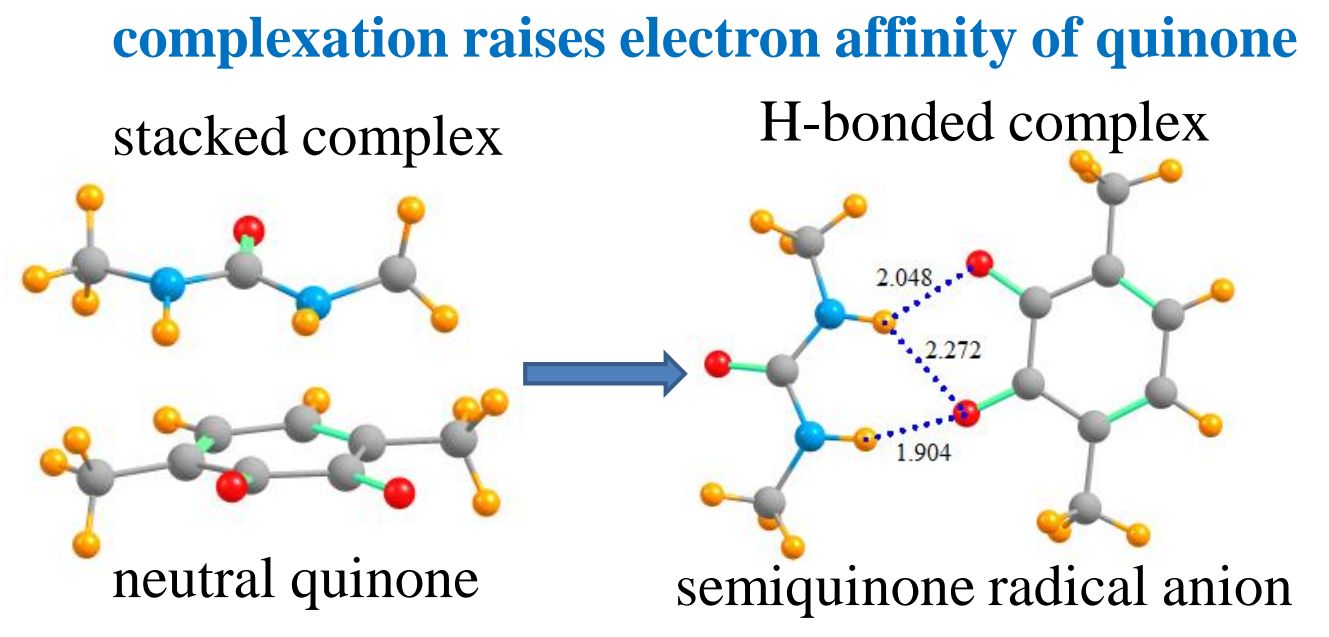

